\title{
ESTRUCTURA COMUNITARIA DEL TAXA FORAMINIFERIDA (PROTOZOA), SEGÚN PROFUNDIDAD, EN EL FIORDO COMAU, CHILOÉ, CHILE.
}

\author{
COMMUNITY STRUCTURE OF FORAMINIFERA (PROTOZOA) TAXA \\ FROM TWO WATER DEPTHS (COMAU FJORD, CHILOÉ, CHILE).
}

Tatiana Hromic ${ }^{1}$

\begin{abstract}
RESUMEN
La composición de las comunidades foraminiferológicas en sedimentos superficiales de aguas menores a $30 \mathrm{~m}$ y mayores a $80 \mathrm{~m}$ del fiordo Comau, golfo de Ancud, Chiloé, Chile, aplicando SIMPER, ANOSIM, MDS y análisis de Cluster, muestran una alta diferencia y distinguen dos comunidades: una de aguas someras, en la cual las especies que más contribuyen a la similitud de las muestras son Ammonia beccarii (87,6 \%) y Buccella peruviana (4,48 \%) y otra de aguas más profundas, en la cual, las especies que más contribuyen a su individualización son: Uvigerina bifurcata $(24,8 \%)$, Bulimna notovata $(21,8 \%)$, Bulimina patagonica (14,42\%), Nonionella auris (10,11\%), Globocassidulina minuta (7,37\%), Nonionoides grateloupi (3,94\%), Quinqueloculina seminula (3,44\%), Buccella frigida $(3,13 \%)$ y Bulimina marginata (2,93\%). Todas ellas eran conocidas en el área de canales y fiordos patagónicos chilenos. Ambas comunidades evidencian también diferencias en relación con el número de especies, abundancia, diversidad y homogeneidad, parámetros que aumentan casi al doble en las aguas más profundas. La cantidad de foraminíferos con caparazón arenáceo tiende a aumentar hacia las profundidades mientras que los porcelanoides tienden a disminuir.
\end{abstract}

Palabras clave: foraminíferos bentónicos, distribución batimétrica

\begin{abstract}
Assemblages of foraminifera were studied at two depths (surface sediment obtained from shallow water $<30 \mathrm{~m}$ and deep water $>80 \mathrm{~m}$ ) in Comau Fjord, Chiloé, Chile. From analyses of results by SIMPER, ANOSIM, MDS, and Cluster Analyses, two distinct communities were described. In the shallow water sediments Ammonia beccarii (87.6\%) and Buccella peruviana (4.48\%) are the species which contributed most to the similarity of the samples. From the deep water sediment, the main species contributing to the internal similarity were: Uvigerina bifurcata (24.8\%), Bulimna notovata (21.8\%), Bulimina patago-

1 Laboratorio de Micropaleontología, Instituto de la Patagonia, Universidad de Magallanes, Punta Arenas, Chile. tatiana. hromic@umag.cl
\end{abstract}


nica (14.42\%), Nonionella auris (10.11\%), Globocassidulina minuta (7.37\%), Nonionoides grateloupi (3.94\%), Quinqueloculina seminula (3.44\%), Buccella frigida (3.13\%) and Bulimina marginata (2.93). All these species are well known in the Chilean channels and fjords area. Benthic foraminifera abundance, species richness, diversity and evenness percentage were almost twice as great in the deep water than the surface water. Abundance of arenaceous foraminifera increased and porcelanceous foraminifera decreased, with water depth.

Key words: Benthic foraminifera, bathymetric distribution.

\section{INTRODUCCIÓN}

La costa chilena, al sur de latitud $42^{\circ} \mathrm{S}$, es una vasta y compleja zona geográfica compuesta de multitud de islas, canales y fiordos formados tras la última glaciación, hace alrededor de 18.000 años. La zona del golfo de Ancud, en la región más septentrional de los canales patagónicos chilenos, es frecuentemente utilizada por las industrias para los cultivos marinos, y esta utilización ha generado gran interés entre ambientalistas y científicos por las consecuencias que podría tener la acumulación de sustancias de desecho en los ecosistemas marinos. Debido al escaso conocimiento que había de la fauna y flora del lugar, las investigaciones sobre biodiversidad se han intensificado en las últimas décadas, destacándose el estudio multidisciplinario realizado en el marco de las expediciones oceanográficas Cimar Fiordo organizadas por el Comité Oceanográfico Nacional del Chile (CONA) en el área de canales y fiordos patagónicos y que ha constituido un valioso aporte al conocimiento de los diversos grupos zoológicos, como crustáceos, poliquetos, peces, etc. (Informes de cruceros Cimar Fiordos (CONA), 1993 a la fecha).

La distribución de los foraminíferos bentónicos depende de una gran variedad de parámetros, entre ellos se puede mencionar temperatura, salinidad, profundidad, disponibilidad de oxígeno, nutrientes y presencia de sustancias tóxicas, entre otros (Colom 1974, Murray 1991, 2000, Jorissen 1999). Las variaciones de dichos parámetros generan microhábitats, que pueden ser favorables para el establecimiento de algunas especies y desfavorables para otras especies y/o comunidades (Jorissen 1999). El conocimiento de la composición faunística que caracteriza a estos microhábitats permite, por una parte, delinear los hábitats y sus parámetros ambientales asociados, en condiciones normales, y por otra parte, analizar las fluctuaciones de los foraminíferos pudiendo, en consecuencia, ser utilizados como indicadores de las variaciones de dichos parámetros. De allí que es posible establecer un referente para estudios posteriores sobre localización y nivel de impacto por acción antrópica, lo que hace que estos microorganismos sean una herramienta útil para estudios ecológicos y de interpretación ambiental.

El objetivo primario de este estudio es documentar y caracterizar las comunidades de foraminíferos bentónicos en los fiordos chilenos de diferentes profundidades, para proveer una línea de base a usar por futuros investigadores como referente y para interpretar futuros cambios comunitarios causados por variaciones ambientales producto de la actividad económica acuícola.

\section{MATERIAL Y MÉTODOS}

\section{Descripción del área}

El fiordo Comau, $\left(42^{\circ} 30^{\prime} S-73^{\circ} 50\right.$ 'W) situado en la costa continental chilena, frente al golfo de Ancud, se origina por una falla reconocida, que se extiende a través de 68 kilómetros en sentido norte-sur. El fiordo alcanza una profundidad máxima de 500 metros con fuertes pendientes laterales. La costa presenta diferentes tipos de sustrato, tales como marismas de lodo, playas de arena, de ripio y bolones; sin embargo, predominan las playas rocosas. Al fiordo desembocan varios cursos de agua y recibe un aporte anual de más de $6.000 \mathrm{ml}$ de aguas lluvia (Fig. 1).

En Comau se pueden distinguir claramente tres capas de agua: a) una capa superficial de alrededor de $10 \mathrm{~m}$, relacionada con los aportes inmediatos de agua dulce, con una baja salinidad $(<10 \%)$ y que aumenta hacia la boca. b) una picnoclina con una alta diferencia de temperatura en verano, y c) 


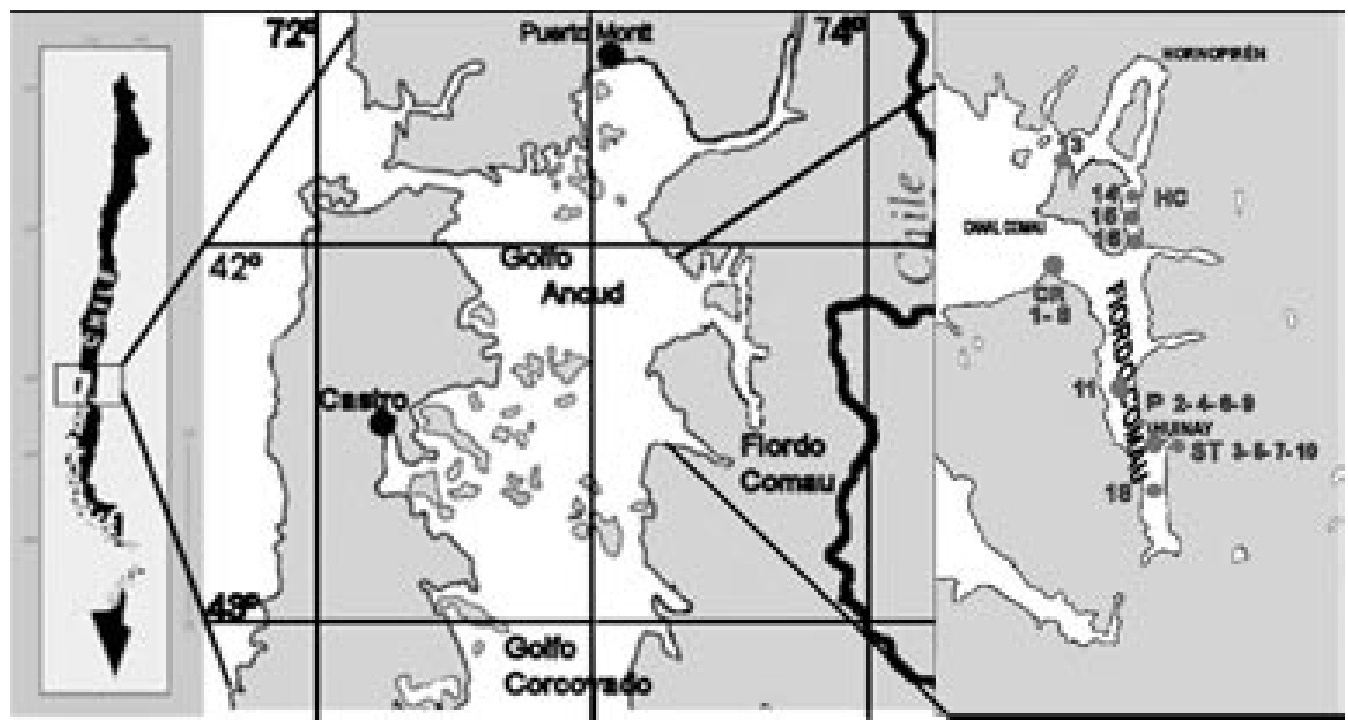

Fig.1: Ubicación de las estaciones en el fiordo Comau, desde donde se extrajeron las muestras. (CR= Caleta del Rey: muestras 1 y $8 ; \mathrm{HC}=$ Hornopirén: muestras 13, 14, 15 y 16; ST= Estación Huinay: muestras 3, 5, 7 y 10; Fiordo Comau: muestras 11 y 12 y P= Punta: muestras 2, 4, 6 y 9).

una capa profunda caracterizada por una salinidad del $32 \%$ y una temperatura de $11^{\circ} \mathrm{C}$. La amplitud máxima de marea es de 7,5 metros. La circulación y el intercambio de agua con el área externa al fiordo están gobernados por procesos estuarinos (Djurfeldt) ${ }^{1}$.

Este ambiente hace que las comunidades bentónicas superficiales sean, en general, más pobres en diversidad de especies y muy distintas de las que se encuentran por debajo de los $10 \mathrm{~m}$ de profundidad. En aguas menores a $10 \mathrm{~m}$ de profundidad, la comunidad está dominada por moluscos mitílidos. A mayor profundidad la comunidad se vuelve más diversa, apareciendo parches de corales de aguas frías que proporcionan albergue tanto a vertebrados como invertebrados. Dentro de ellos los gorgónidos son frecuentes actuando como sustrato para diferentes tipos de hydrozoos y bryozoos ${ }^{2}$.

No se observan glaciares en la boca del fiordo, provenientes del Campo Patagónico de Hielo Norte, como sucede en fiordos más australes.

\section{Material y trabajo de laboratorio}

Se revisaron 16 muestras de sedimentos marinos superficiales provenientes del fiordo Comau, 1,3 http: // www.huinay.cl
Chiloé. Diez de ellas fueron obtenidas mediante buceo autónomo en tres transectos (Punta, Estación y caleta del Rey) desde profundidades que variaron entre 8 y 30 m. Las otras 6 muestras se obtuvieron durante los cruceros Cimar Fiordos $N^{\circ} 10$ y $N^{\circ} 11$ organizados por el Comité Oceanográfico Nacional (CONA), desde profundidades superiores a $80 \mathrm{~m}$ (Fig.1 y Tabla 1)

El material fue lavado usando un tamiz de 63 micrómetros y secado a temperatura ambiental. Los foraminíferos totales (vivos y muertos) se extrajeron uno a uno bajo lupa binocular, desde $50 \mathrm{~g}$ de material o de la cantidad de sedimento disponible de cada muestra. Se estandarizaron los valores a ejemplares por gramo de sedimento seco. Los ejemplares fueron pegados en reglillas por especie y contados (Boltovskoy 1965). Para la determinación hasta el nivel genérico se siguió a Loeblich \& Tappan (1988) y Decrouez (1989). Para el nivel específico a Brady (1884), Barker (1960) y la literatura del área (Boltovskoy et al. 1980, Hromic 1996, 1998, 1999, 2001, 2002, 2003, 2008, Hromic \& Zúñiga 2005, Hromic et al. 2006, Marchant 1993, Zapata \& Moyano 1997). Para determinar las especies con mayor distribución geográfica en el fiordo se estableció como límite la presencia de la especie en al menos el $50 \%$ de las muestras. 
TABLA 1: Estaciones en que se recolectaron muestras: ubicación, profundidad y modo de obtención.

\begin{tabular}{|c|c|c|c|c|}
\hline No muestra & Estación & Ubicación & Profundidad (m) & $\begin{array}{c}\text { Modo de extracción } \\
\text { de la muestra }\end{array}$ \\
\hline 1 & CR-8 & Caleta del Rey & 8 & Buceo \\
\hline 2 & P-10 & Punta & 10 & Buceo \\
\hline 3 & ST-10 & Estación & 15 & Buceo \\
\hline 4 & P-15 & Punta & 15 & Buceo \\
\hline 5 & ST-15 & Estación & 20 & Buceo \\
\hline 6 & P-20 & Punta & 20 & Buceo \\
\hline 7 & ST-20 & Estación & 20 & Buceo \\
\hline 8 & CR-20 & Caleta del Rey & 25 & Buceo \\
\hline 9 & P-25 & Punta & 30 & Buceo \\
\hline 10 & ST-30 & Estación & 350 & Draga Mclntyre \\
\hline 11 & St 18 (Cimar 10) & Comau & 220 & Draga Mclntyre \\
\hline 12 & St 19 (Cimar 10) & St HC 3 (Cimar 11) & Hornopirén.Comau & 158 \\
\hline 13 & St HC 5 (Cimar 11) & Hornopirén-Comau & 108 & Draga McIntyre \\
\hline 14 & St HC 6 (Cimar 11) & Hornopirén-Comau & 483 & Draga McIntyre \\
\hline 15 & St HC 8 (Cimar 11) & Hornopirén-Comau & & Draga McIntyre \\
\hline 16 & & & & \\
\hline
\end{tabular}

\section{Análisis estadísticos}

Las diferencias y similitudes entre las asociaciones de foraminíferos de los dos niveles de profundidad (somero y profundo) se estableció mediante métodos multivariados de ordenación y clasificación. Mediante el análisis SIMPER se investigó la diferencia entre las asociaciones de aguas someras y de aguas profundas, y la similitud entre las muestras internas de cada grupo. Se utilizó el análisis ANOSIM para establecer diferencias significativas. Posteriormente se aplicó el método del escalamiento multidimensional (MDS) siendo corroborado por análisis jerárquico de conglomerados (índice de Bray-Curtis), para evaluar la separación de los grupos. También se calculó el índice de diversidad Shannon-Wiener y el de equidad (Pielou), todos incluidos en el programa PRIMER 5.0 (Carr 1997, Clarke \& Warwick 2001).

\section{RESULTADOS}

\section{Sistemática}

Se determinó un total de 66 especies de foraminíferos bentónicos las que se clasificaron en 5 subórdenes, 27 familias y 38 géneros en las 16 muestras analizadas (Fig. 2). El suborden con mayor número géneros y especies fue Rotaliina.

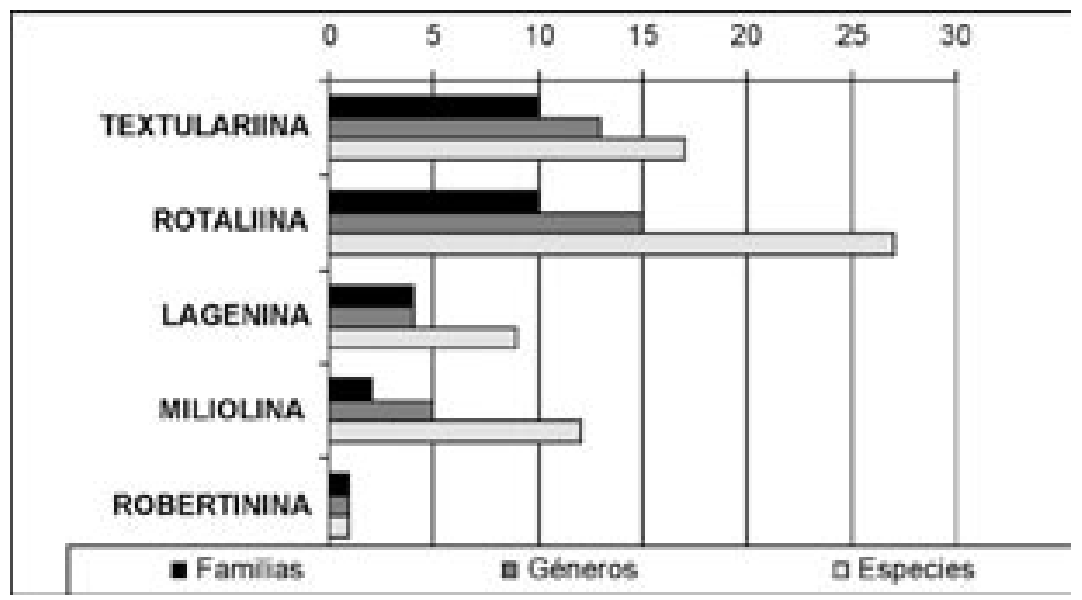

Fig. 2: Distribución de familias, géneros y especies según suborden 


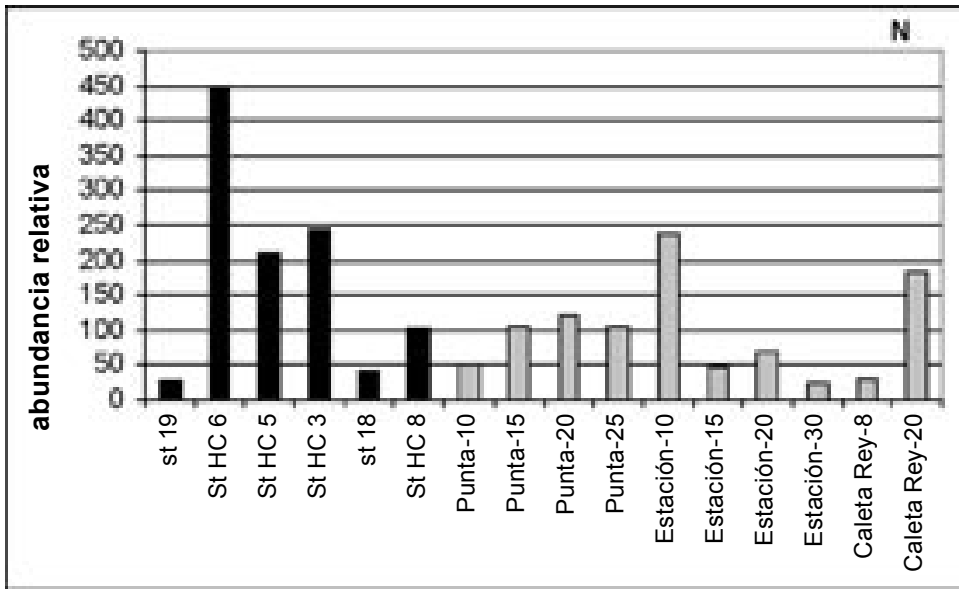

Fig. 3: Abundancia de foraminíferos bentónicos en el fiordo Comau, Chiloé, Chile. En negro las muestras obtenidas en aguas de más de 10 metros de profundidad y en gris, las muestras pertenecientes a aguas más someras.

\section{Abundancia}

Se recolectó un total de 2.044 ejemplares, 1.069 de ellos fueron extraídos de aguas profundas y 975 de aguas someras. Expresados como número de ejemplares promedio por gramo de muestra, se observa una gran diferencia de la abundancia: en aguas someras se aprecia prácticamente la mitad del promedio de ejemplares que en profundidad (Figs. 3 y 8 ).

Sin embargo no se observa una clara tendencia a aumentar el número de ejemplares en aguas profundas (R2=0,4075) (Fig. 4).

\section{Riqueza específica}

De las 66 especies (vivas más muertas) que se determinaron en el fiordo Comau, 44 especies habitan en aguas profundas y 34 en aguas someras. Del total, 11 especies fueron comunes. De las 34 especies determinadas en ambientes someros, sólo 4 presentaron una frecuencia superior al 50\%: Ammonia beccarii que se encontró en el 90\% de las muestras y con una abundancia total del 63,2\%; en el 70\% de las muestras se presentó Quinqueloculina seminula, (abundancia: 12,1\%), y Buccella peruviana (7,1\% de abundancia). En cambio, Cibicides fletcheri, a pesar de tener una escasa presencia (2,9\%), se presentó en el 50\% de las muestras. Las especies

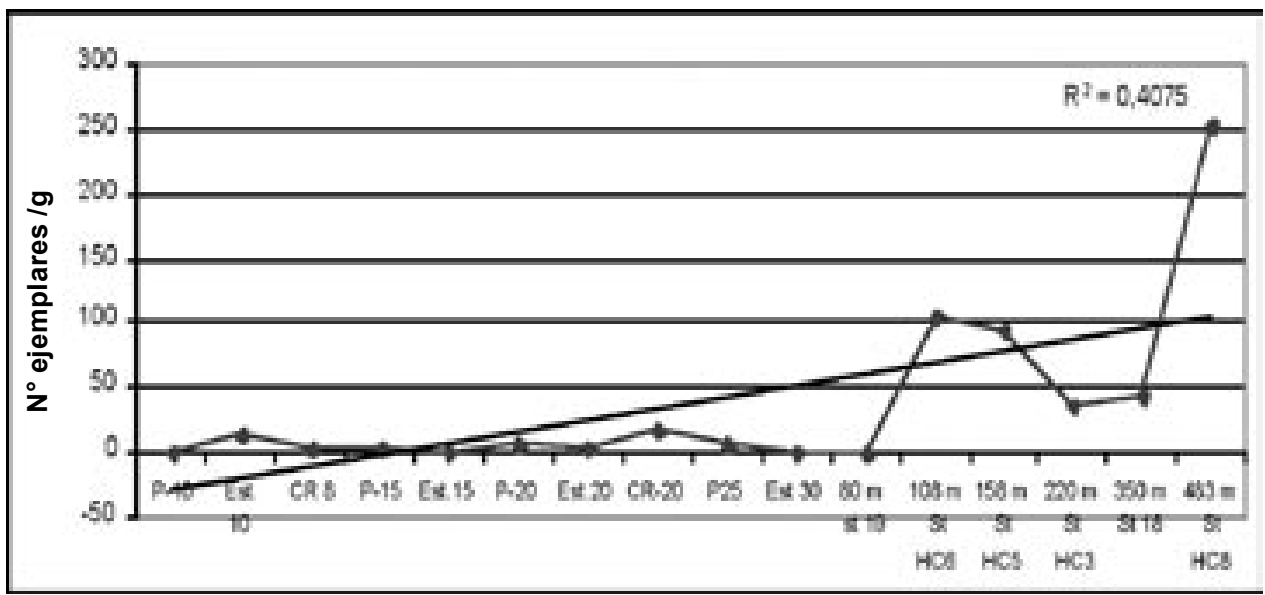

Fig. 4: Abundancia de foraminíferos bentónicos (ejs/g), según la profundidad. Las muestras se encuentran ordenadas de menor a mayor profundidad. 
TABLA 2: Lista de especies de foraminíferos bentónicos con número de ejemplares, abundancia relativa y porcentaje de frecuencia en las aguas más profundas, del fiordo Comau, golfo de Ancud, Chiloé, Chile.

\begin{tabular}{|l|c|c|c|c|}
\hline \multicolumn{1}{|c|}{ Especie } & N & $\%$ & Frecuencia & $\%$ \\
\hline Buccella frigida & 21 & 1,96 & 5 & 83,33 \\
\hline Nonionella auris & 83 & 7,76 & 5 & 83,33 \\
\hline Bulimina notovata & 97 & 9,07 & 5 & 83,33 \\
\hline Quinqueloculina seminula & 32 & 2,99 & 4 & 66,67 \\
\hline Uvigerina bifurcata & 168 & 15,72 & 4 & 66,67 \\
\hline Cibicides dispars & 9 & 0,84 & 4 & 66,67 \\
\hline Globocassidulina minuta & 115 & 10,76 & 4 & 66,67 \\
\hline Bulimina aculeata & 49 & 4,58 & 3 & 50,00 \\
\hline Bulimina marginata & 38 & 3,55 & 3 & 50,00 \\
\hline Alveolophragmiun wiesneri?? & 6 & 0,56 & 3 & 50,00 \\
\hline Bulimina patagonica & 158 & 14,78 & 3 & 50,00 \\
\hline Cassidulinoides parkerianus & 32 & 2,99 & 3 & 50,00 \\
\hline Cibicides refulgens & 5 & 0,47 & 3 & 50,00 \\
\hline Eggerella scabra & 12 & 1,12 & 3 & 50,00 \\
\hline Lagena leavis & 3 & 0,28 & 3 & 50,00 \\
\hline Nonionoides grateloupi & 108 & 10,10 & 3 & 50,00 \\
\hline
\end{tabular}

restantes se distribuyeron con una frecuencia igual o inferior al $40 \%$ de las muestras.

En ambientes más profundos, $>80 \mathrm{~m}$, de las 44 especies encontradas, 16 presentaron una distribución superior al $50 \%$ de las muestras. Las especies con mayor frecuencia se indican en la tabla 2. En términos de abundancia, las especies más representadas fueron Uvigerina bifurcata, Bulimina patagonica, Globocassidulina minuta, y Globobulimina notovata.

La riqueza de especies promedio por ambiente representada en la figura 8 , destaca que el número promedio de especies totales se duplica en la zona más profunda (15 especies promedio) en relación con la zona somera (7,5 especies).

En relación con el número de especies por muestras, las estaciones HC 6 (muestra 15) con 23 especies, caleta del Rey-20 (muestra 8) con 20 especies, y HC 5 (muestra 14) con 19 especies, poseen la mayor riqueza de especies (Fig. 5).

\section{Diversidad}

Los valores de diversidad oscilaron entre 0,000 en la estación Punta-10 y 1,93 en la estación caleta Rey-20, en la zona somera. En la zona más

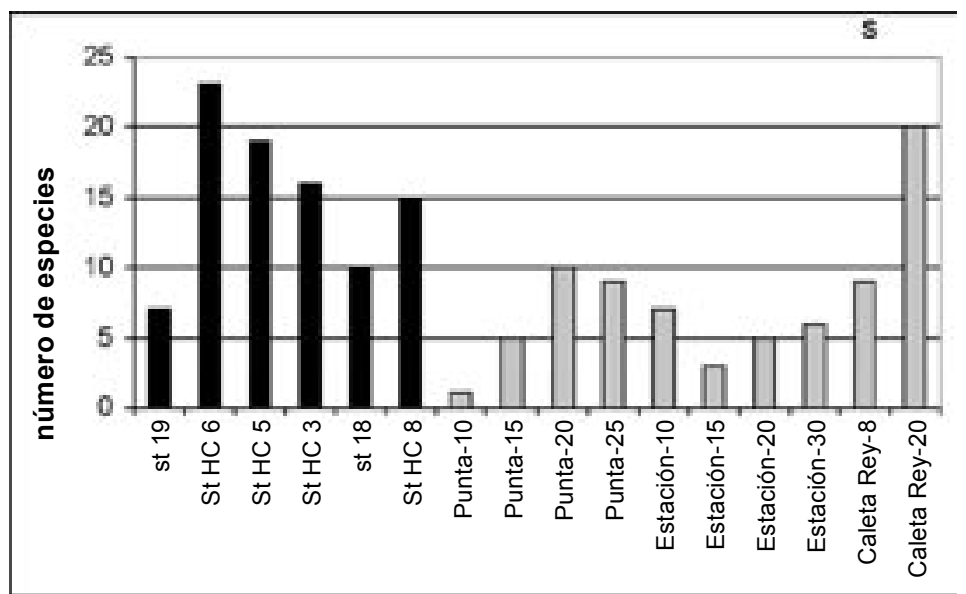

Fig. 5: Número de especies recolectadas en cada una de las muestras. En negro las muestras obtenidas en aguas de más de 10 metros de profundidad y en gris, las muestras pertenecientes a aguas más someras. 


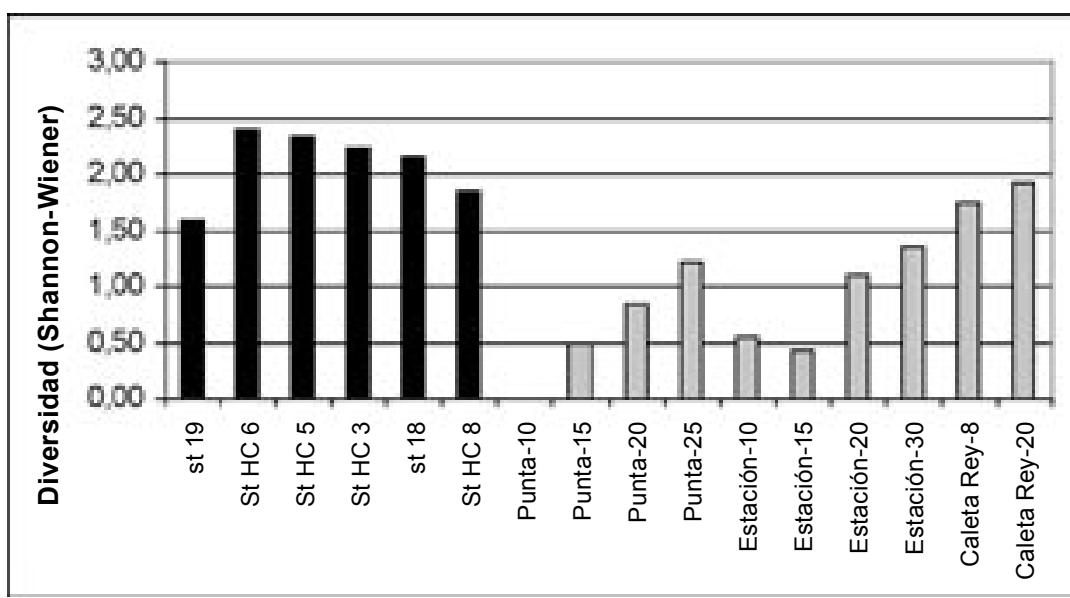

Fig. 6: Valores de diversidad en cada muestra obtenidos con el índice de Shannon-Wiener. En negro las muestras obtenidas en aguas de más de 10 metros de profundidad y en gris, las muestras pertenecientes a aguas más someras.

profunda los valores estuvieron entre 1,59 y 2,39, en promedio se duplica en la zona más profunda (Figs. 6 y 8).

\section{Equidad}

El índice de Pielou supera el valor de 0,65 en aguas profundas mostrando un mayor número de especies contribuyentes a la abundancia total $y$ es inferior a 0,8 en aguas más someras, con una mayor dominancia de las especies (Fig. 7).

En promedio las estaciones de agua profunda mostraron casi el doble de homogeneidad que en aguas someras, $e$ indican una mayor dominancia de especie en aguas someras (Fig. 8).

\section{Comparación}

En aguas someras, Ammonia beccarii es claramente la especie dominante. Se presentó solitaria en la muestra 2 , estación Punta a $10 \mathrm{~m}$ de profundidad, en donde se recolectaron 52 ejemplares y muy abundante en la muestra 5 , Estación a $15 \mathrm{~m}$ de profundidad (>50\%). En Estación a 10 y 20 m de profundidad, fue acompañada por Buccella peruviana. De acuerdo con los resultados del análisis SIMPER, el porcentaje de similitud entre las muestras que conformaron el grupo de aguas someras es el 32,19\%; las especies que caracterizarían los ambientes someros se listan en la tabla 3.

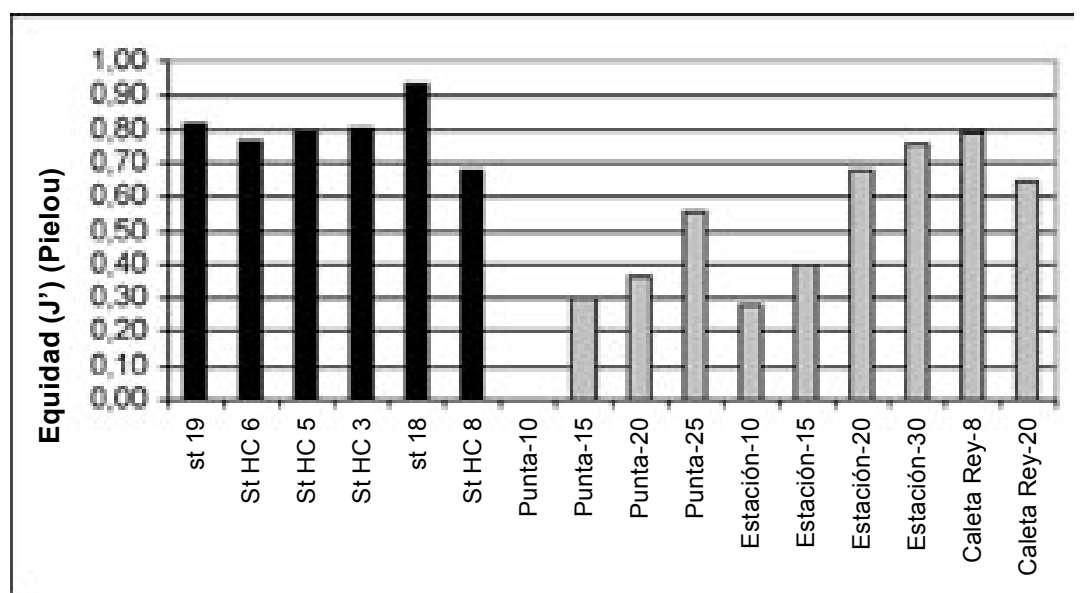

Fig. 7: Valores de equidad (Pielou) obtenidos en cada una de las muestras del Fiordo Comau. En negro las muestras obtenidas en aguas de más de 10 metros de profundidad y en gris, las muestras pertenecientes a aguas más someras. 


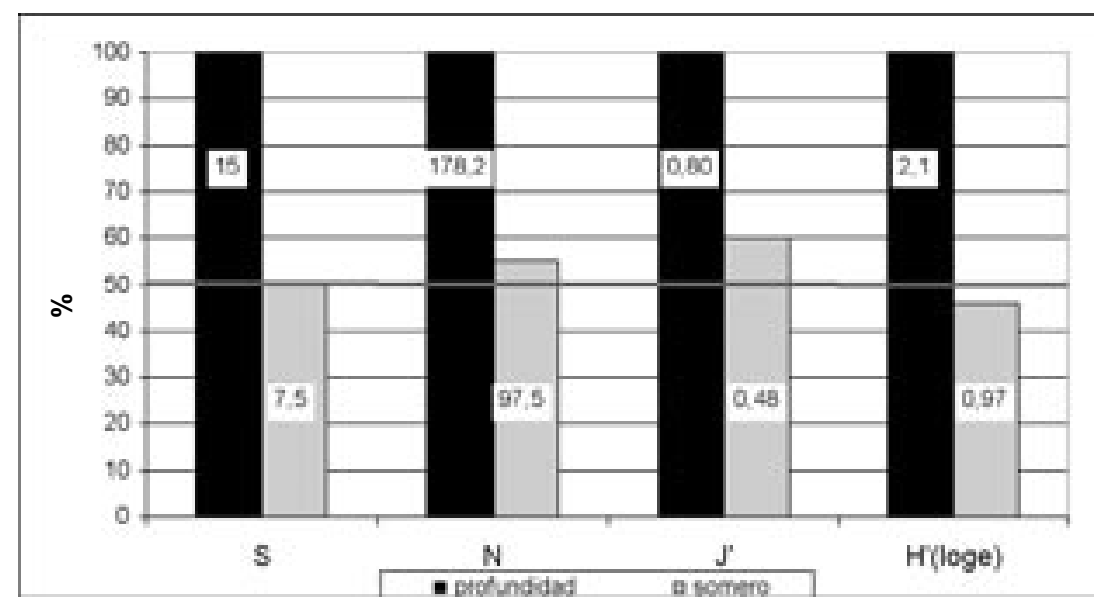

Fig. 8: Relación porcentual del promedio del número de especies (S), abundancia (N) (ejs/g), equidad (J') y diversidad entre la zona profunda $(>80 \mathrm{~m}=$ negro)) y zona somera $(<30 \mathrm{~m}=$ gris $)$ en fiordo Comau. La línea roja indica el $50 \%$ de la relación.

En aguas más profundas la homogeneidad aumentó; suele encontrarse entre 2 y 4 especies dominantes por muestra no siendo siempre las mismas. En la muestra 11 dominaron $B$. marginata, $Q$. seminula y $T$. trigonula, y en la estación HC-3 B. aculeata y B. patagonica, G. minuta y $U$. bifurcata.

En las muestras 11,12 y 16 , dominaron $B$. patagonica, $U$. bifurcata y $B$. notovata respectivamente. Finalmente en la muestra 15 , las especies dominantes fueron $B$. patagonica y $G$. minuta, $N$. grateloupi y $U$. bifurcata.
El porcentaje de similitud entre las muestras del grupo de aguas profundas fue más bajo (20,09\%) que el de aguas someras. Nueve especies contribuyeron al $90 \%$ de disimilitud. En la tabla 4 se muestran las especies que caracterizan el ambiente profundo con los resultados del SIMPER.

Paralelamente SIMPER evidenció un 97,21\% de disimilitud entre la composición foraminiferológica del sector somero y profundo, en la que las 7 especies que más contribuyen a la disimilitud se muestran en la tabla 5.

TABLA 3: Especies que caracterizan al ambiente somero, con sus promedios de abundancia (PA) y de similitud (PS); desviación estándar (SIM/DE); el porcentaje de contribución (\%CONT) a las diferencias con el grupo de ambientes profundos y el porcentaje de contribución acumulado (CONT A).

\begin{tabular}{|c|c|c|c|c|c|}
\hline Especies & PA & PS & SIM/DE & \% CONT & \% CONT A \\
\hline Ammonia beccarii & 61,60 & 28,21 & 1,06 & 87,64 & 87,64 \\
\hline Buccella peruviana & 6,90 & 1,44 & 0,51 & 4,48 & 92,12 \\
\hline
\end{tabular}

TABLA 4: Especies que caracterizan el ambiente profundo del fiordo Comau, con sus promedios de abundancia (PA); promedio de similitud (PS); desviación estándar (SIM/DE); el porcentaje de contribución (\% CONT) a las diferencias con el grupo de ambientes profundos y el porcentaje de contribución acumulado (\% CONT A).

\begin{tabular}{|c|c|c|c|c|c|}
\hline Especie & PA & PS & SIM/DE & \% CONT & \% CONT A \\
\hline Uvigerina bifurcata & 28,00 & 4,98 & 0,64 & 24,80 & 24,80 \\
\hline Bulimina notovata & 16,17 & 4,38 & 1,10 & 21,81 & 46,61 \\
\hline Bulimina patagonica & 26,33 & 2,90 & 0,47 & 14,42 & 61,03 \\
\hline Nonionella auris & 13,83 & 2,03 & 0,76 & 10,11 & 71,15 \\
\hline Globocassidulina minuta & 19,17 & 1,48 & 0,56 & 7,37 & 78,51 \\
\hline Nonionoides grateloupi & 18,00 & 0,79 & 0,47 & 3,94 & 82,46 \\
\hline Quinqueloculina seminula & 5,33 & 0,69 & 0,55 & 3,44 & 85,90 \\
\hline Buccella frigida & 3,50 & 0,63 & 0,98 & 3,13 & 89,03 \\
\hline Bulimina marginata & 6,33 & 0,59 & 0,43 & 2,93 & 91,96 \\
\hline
\end{tabular}


TABLA 5: Especies del fiordo Comau, Chile, que más contribuyeron a las diferencias entre las asociaciones de foraminíferos de aguas someras y aguas profundas. Se muestra promedio de abundancia (PA) del grupo 1 (aguas profundas) y grupo 2 (aguas someras); promedio de disimilitud (PD); disimilitud y desviación estándar (DISS/DE); el porcentaje de contribución (\% CONT) y porcentaje de contribución acumulado (\% CONT A).

\begin{tabular}{|l|c|c|c|c|c|c|}
\hline \multicolumn{1}{|c|}{ Species } & Grupo 1 & Grupo 2 & & & & \\
\hline Ammonia beccarii & PA & PA & PD & DISS/DE & \%CONT & \%CONT A \\
\hline Uvigerina bifurcata & 0,00 & 61,60 & 26,02 & 1,15 & 26,77 & 26,77 \\
\hline Bulimina patagonica & 28,00 & 00,00 & 9,53 & 0,91 & 9,81 & 36,58 \\
\hline Bulimina notovata & 26,33 & 00.00 & 7,12 & 0,88 & 7,32 & 43,90 \\
\hline Quinqueloculina seminula & 16,17 & 0,20 & 5,98 & 1,36 & 6,16 & 50,05 \\
\hline Globocassidulina minuta & 5,33 & 11,80 & 5,06 & 0,59 & 5,21 & 55,26 \\
\hline Nonionella auris & 19,17 & 0,50 & 4,71 & 0,92 & 4,84 & 60,10 \\
\hline
\end{tabular}

Estos resultados concuerdan con los obtenidos al analizar las muestras con ANOSIM ( $\mathrm{R}$ global= $0,821, \mathrm{P}<0,1 \%$ ) y con MDS (Fig. 9) que señala claramente la conformación de dos grupos, como se observa en el dendrograma (Fig. 10).

\section{Relación calcáreo/arenáceo}

Los foraminíferos con caparazón calcáreo hialino particularmente del suborden Rotaliina, fueron los más abundantes en las muestras respecto del número de ejemplares, así como de especies en todas las profundidades. Los organismos con caparazón arenáceo, en general bastantes más escasos, aumentaron hacia las profundidades y los calcáreos porcelanoides aumentaron en las aguas someras (Fig.11).

\section{DISCUSIÓN}

La distribución de foraminíferos en canales y fiordos patagónicos se debe a la conjunción de muy diversos factores cuyo entendimiento resulta ser un gran desafío. Entre estos factores se encuentran la heterogeneidad de hábitats y sus respectivas asociaciones y que están determinados por la gran variabilidad en sustratos, las fuertes corrientes mareales, las diversas fisiografías y, en general, por la influencia de factores locales como la cercanía de los Campos de Hielo Norte y Sur (Violanti et al. 2000, Hromic et al. 2006). En aguas australes, los trabajos se han focalizado en dar a conocer la composición faunística y algunos intentos de distribución espacial pero la problemática de la distribución batimétrica de los foraminíferos se ha abordado escasamente. Se conoce un intento de

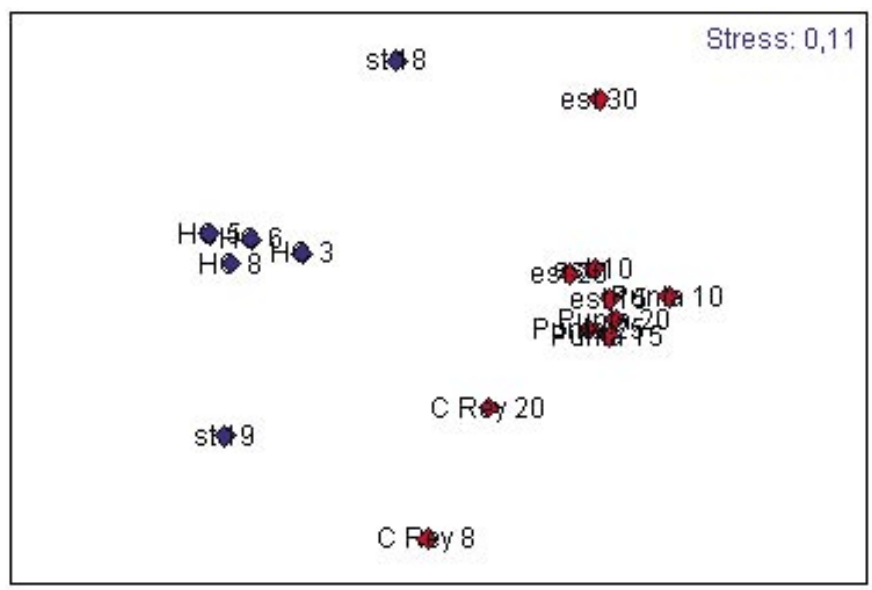

Fig. 9: Ordenamiento MDS con datos de abundancia de especies de foraminíferos bentónicos de las muestras de aguas profundas (azul) y aguas someras (rojo). 


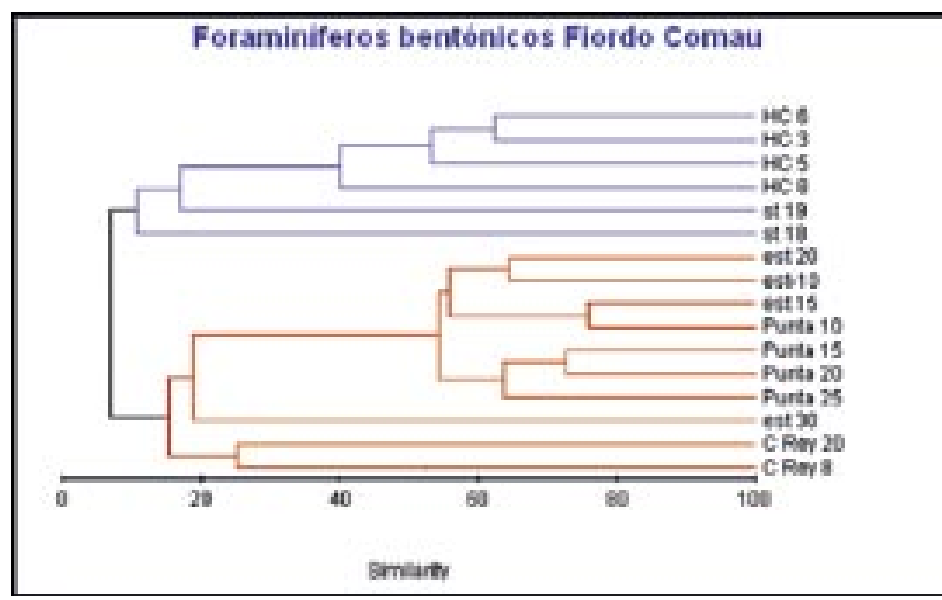

Fig. 10: Análisis de conglomerados utilizando el índice de similitud de Bray-Curtis que evidencia la conformación de dos grupos de muestras, de aguas profundas (azul) y de aguas someras (rojo).

zonación batimétrica al sur del estrecho de Magallanes (Hromic 2009), en donde se reconocieron 3 tramos: menor de $150 \mathrm{~m}$, entre 150 y 350 m y mayor de 350 $\mathrm{m}$. En dicho trabajo, en el tramo más somero (0-50 $\mathrm{m})$ se encontraron: Buccella frigida, Buliminella elegantisima y Discorbis bertheloti, destacándose la ausencia de Ammonia beccarii. En los tramos más profundos se recolectaron especies similares a las encontradas en el del fiordo Comau como Bulimina notovata y Nonioides grateloupi pero en cambio no se presentaron Uvigerina bifurcata, Bulimina patagonica y Nonionella auris, comunes al sur del estrecho de Magallanes. Una investigación llevada a cabo en el Área Marina Costera Protegida (AMCP) Francisco Coloane sobre el estrecho de Magallanes (Hromic
$2008^{3}$ ) y que analizó los foraminíferos presentes hasta los $20 \mathrm{~m}$ de profundidad, dejó en evidencia que entre las especies indicadoras se encuentran Ammonia beccarii y Buccella peruviana entre otras, de lo cual se desprende que estas especies son reconocidas como componentes principales de las asociaciones presentes en sedimentos someros. Hromic \& Zúñiga (2005) también encontraron Ammonia beccarii en aguas someras entre $44^{\circ}$ y $46^{\circ} \mathrm{S}(7-12 \mathrm{~m})$ en forma abundante (28,1\%), junto a Buccella frigida (17,7\%)

3 Hromic, T. 2008 Foraminíferos bentónicos como indicadores biológicos de ambientes costeros, del Área Marina Costera Protegida Francisco Coloane, Magallanes, Chile. XXVIII Congreso de Ciencias del Mar, Viña del Mar, Chile, Mayo, 2008.

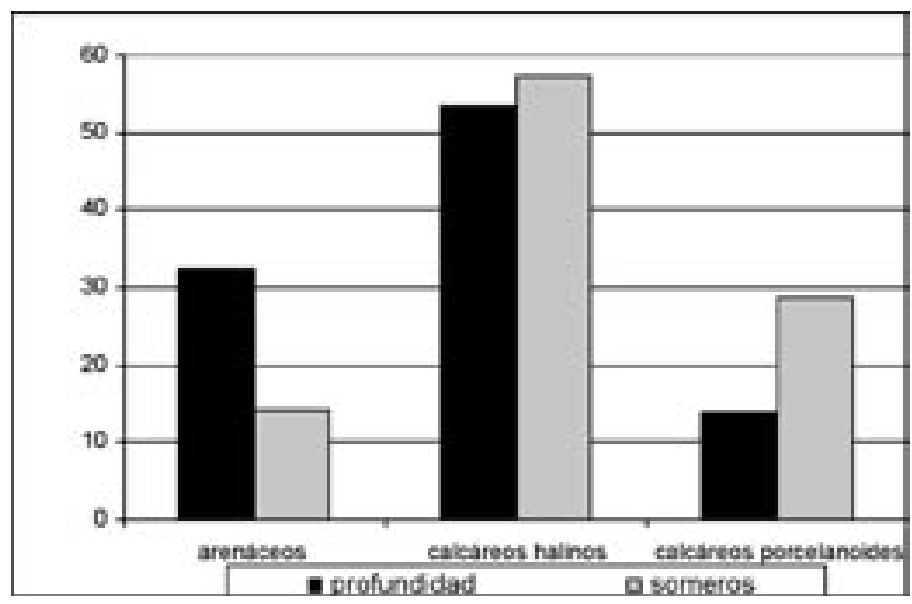

Fig. 11: Distribución de los organismos con caparazón arenáceo, calcáreo hialino y calcáreo porcelanoide según profundidad, en el fiordo Comau 
y Cibicides dispars (14,8\%). Esta especie se encontró viva, además, en la costa de bahía Maullín entre 0-3 m (Zapata \& Varela 1975).

Según Murray (1991, 2000) Ammonia beccarii es una especie cosmopolita que caracteriza ambientes costaneros, de aguas someras y de condiciones eurihalinas, por lo cual los resultados de este estudio concuerdan con otras investigaciones. Sin embargo, en bahía Scholl (Marchant 1993), no aparece Ammonia beccarii, siendo abundantes en cambio, Cribrorotalia meridionalis (45,1\%), Cibicides aknerianus (24,8\%) y Elphidium macellum (12,5\%) ausentes en Comau. Tampoco aparece mencionada para el estrecho de Magallanes (Zapata \& Alarcón 1988), lo que podría relacionarse con un ambiente de menor energía en el fiordo. Ishman \& Martínez (1995) afirman que las variedades de Cibicides son propios de ambiente de alta energía. Lena (1966) analizó muestras de sedimento costaneros extraídos desde $9 \mathrm{~m}$ de profundidad en el canal Beagle, no encontrando tampoco Ammonia beccarii pero sí, abundantes Buccella frigida, mencionando que esta última es una especie común, en la costa argentina en donde llega hasta los $80 \mathrm{~m}$. Encontró también, en forma abundante Cibicides aknerianus, Elphidium macellum s.l. y Globocassidulina. crassa, que en Comau no se registraron, concordando con los resultados de Marchant (1993).

Violanti et al. (2000) señalaron que las globocassidulinas son frecuentes en ambientes de baja productividad y que B. peruviana (s.l) es frecuente en ambientes someros con arena gruesa. La presencia de abundantes formas arenáceas al interior del fiordo en las partes más profundas es consistente con los datos entregados por Jennings \& Helgadottir (1994) y Korsun \& Hald (2000) para fiordos del hemisferio norte, en donde atribuyen este patrón a la baja concentración de calcio por la alta productividad y avance de glaciares, posiblemente aquí esté más relacionado con la alta pluviosidad y aportes continentales de agua dulce que tienden a disminuir la salinidad y en consecuencia a las formas calcáreas.

\section{CONCLUSIONES}

a) En un total de 2.004 ejemplares de foraminíferos bentónicos recolectados se identificaron 66 especies, clasificadas en 5 subórdenes, 27 familias y 38 géneros. 34 de ellas habitaban en aguas someras $(<30 \mathrm{~m})$ y 44 en aguas profundas (>80 m). Se encontraron 11 especies con rango de distribución amplio, i.e. tanto en las aguas someras como en las profundas.

b) Se identificó una asociación de foraminíferos de aguas someras, según análisis SIMPER las especies que más contribuyeron al agrupamiento son: Ammonia beccarii $(87,64 \%)$ y Buccella peruviana $(4,48 \%)$.

c) Se definió una asociación de aguas profundas, según SIMPER las especies con mayor contribución al grupo son: Uvigerina bifurcata (24,80\%), Bulimina notovata (21,81\%), Bulimina patagonica $(14,42 \%)$, Nonionella auris $(10,11 \%)$, Globocassidulina minuta (7,37\%), Nonionoides grateloupi (3,94\%), Quinqueloculina seminula $(3,44 \%)$, Buccella frigida $(3,13 \%)$ y Bulimina marginata $(2,93 \%)$.

d) Las diferencias entre las asociaciones de agua somera y profunda, según SIMPER, estuvieron dadas por Ammonia beccarii (26,77\%), Uvigerina bifurcata (9,81\%), Bulimina patagonica (7,32\%), Bulimina notovata (6,16\%), Quinqueloculina seminula (5,21\%), Globocassidulina minuta (4,84\%), y Nonionella auris (4,24\%). Estas diferencias fueron respaldadas con ANOSIM ( $R$ global $=0,821, P<$ 0,1\%), MDS y Análisis de Cluster.

e) Los promedios de los valores de número de especies $(\mathrm{S})$, abundancia $(\mathrm{N})$, equidad (J') y diversidad $\left(\mathrm{H}^{\prime} \log _{e}\right)$ prácticamente se doblaron en las asociaciones de aguas profundas, al igual que el número de ejemplares con caparazón arenáceo.

\section{AGRADECIMIENTOS}

La autora agradece a la Dra. Vreni Hausserman y al Dr. Günter Fonsterra la extracción y el envío de las muestras de ambientes someros del fiordo Comau. Al Comité Oceanográfico Nacional (CONA) la obtención de muestras profundas, a la Universidad de Magallanes por su apoyo y a la ayudante del Laboratorio de Micropaleontología del Instituto de la Patagonia, Universidad de Magallanes, Srta. Lyta Quezada su dedicación. 


\section{LITERATURA CITADA}

Barker, R.W. 1960. Taxonomic Notes on the Species figured by H. B. Brady in his Report on the Foraminifera dredged by H.M. S. Challenger during the years 1873-1876. Soc. Econ. Paleontol. \& Mineral Sp. Pub. $\mathrm{N}^{\circ}$ 9, Oklahoma, USA.

Boltovskoy, E. 1965. Los Foraminíferos Recientes. Eudeba. Bs. Aires. 510 pp.

Boltovskoy, E., G. Giussani, S. Watanabe \& R. Wright 1980. Atlas of benthic shelf foraminifera of the southwest Atlantic Junk. bv. Pub. The Hague- Boston-London. 147 pp.

Brady, H.B. 1884. Report on the Foraminifera dredged by H.M.S. "Challenger" during the years 1873 -1876. Report Voyage Challenger, Zool., 9: 1 - 814.

Carr, M. R. 1997. Primer User Manual. Plymouth Marine Laboratory Plymouth Routines in Multivariate Ecological Research. Plymouth. PL13DH, UK. 63 pp.

Clarke, K. R. \& R. M. Warwick 2001. Changes in Marine Communities: an aproach to statistical analysis and interpretation $2^{\text {nd }}$ edition Plymouth Marine Laboratory Plymouth Routines in Multivariate Ecological Research. Plymouth.

Colom, G. 1974. Foraminíferos ibéricos. Introducción al estudio de las especies bentónicas recientes. Investigación Pesquera, Consejo Superior de Investigaciones Científicas, vol. 38. Barcelona. $245 \mathrm{pp}$.

Decrouez, D. 1989. Generic ranges of Foraminiferida. Revue Paleobiologie 8 (1): 263 - 321.

Hromic, T. 1996. Foraminíferos bentónicos (Protozoa: Foraminiferida) de aguas profundas del estrecho de Magallanes, Chile. Anales Instituto Patagonia Serie Cs. Nat. (Chile) 24: $65-86$

Hromic, T. 1998. Foraminiferida: Miliolina del estrecho de Magallanes, Chile; distribución en el área subantártica y antártica. Anales Instituto Patagonia Serie Cs. Nat. (Chile) 26: 107-118.

Hromic, T. 1999. Foraminíferos bentónicos de canales australes de Kirke: canal Kirke, golfo Almte. Montt y seno Última Esperanza, XII ${ }^{a}$
Región, Magallanes y Antártica chilena. Anales Instituto Patagonia Serie Cs. Nat.(Chile) 27: 91-104.

Hromic, T. 2001. Foraminíferos bentónicos del canal Baker ( $47^{\circ} \mathrm{S} ; 7^{\circ} \mathrm{W}$ ) Pacífico sudoriental, Chile. Anales Instituto Patagonia Serie Cs. Nat. (Chile) 29: 135 -156.

Hromic, M. T. 2002. Foraminíferos bentónicos de bahía Nassau, Cabo de Hornos, Chile. Comparación con foraminíferos del cono sur de América, antártica e islas Malvinas. Anales Instituto Patagonia Serie Cs. Nat. (Chile) 30: 95-108.

Hromic M. T. 2003. Presencia de foraminíferos bentónicos en canales australes $\left(43^{\circ}-47^{\circ} \mathrm{S}\right)$, resultados preliminares Expedición CIMARFIORDO VIII (2002). Informe de crucero; Revista Comité Oceanográfico Nacional,CONA: 193-200, Valparaíso, Chile

Hromic, T., S. Ishman \& N. Silva 2006. Benthic foraminiferal distributions in Chilean fjords: $47^{\circ} \mathrm{S}$ to $54^{\circ} \mathrm{S}$ Marine Micropaleontology: 115-134.

Hromic, T. \& M. Zúñiga-Rival 2005. Foraminíferos bentónicos de ambientes someros extraídos durante la expedición Cimar 7 fiordos, canales patagónicos $\left(42^{\circ}-47^{\circ} \mathrm{S}\right)$ Boletín de la Sociedad de Biología de Concepción, Chile 76: $25-38$.

Hromic, T. 2009. Distribución batimétrica de foraminíferos bentónicos (Protozoa: Foraminiferida) al sur del estrecho de Magallanes $\left(52^{\circ}-56^{\circ} \mathrm{S}\right)$, Chile. Anales Instituto Patagonia (Chile) 37(1): 23-38.

Ishman, S. \& R. Martínez 1995. Distribution of modern benthic foraminifers from the fjord region of southern Chile. ( $42^{\circ} \mathrm{S}$ to $55^{\circ} \mathrm{S}$ ) Antarctic Journal Rev. 6-8

Jennings A.E. \& G. Helgadottir 1994. Foraminiferal assemblages from the fjords and shelf of eastern Greenland. Journal of Foraminiferal Research 24: 124-144.

Jorissen, F.J. 1999. Benthic foraminiferal microhabitats below the sediment-water interface. In: Gupta, S. (Ed.), Modern Foraminifera. Kluwer Academic Publishing, Dordrecht, pp. 161- 179.

Korsun, S. \& M. Hald 2000. Seasonal dynamics of benthic foraminifera in a glacially fed fjord of 
Svalbard, European Arctic. Journal of Foraminiferal Research. 30: 251-271

Lena, H. 1966. Foraminíferos recientes de Ushuaia (Tierra del Fuego, Argentina). Ameghiniana 4 (9): 311- 336.

Loeblich, A. \& H. Tappan 1988. Foraminiferal Genera and Their Classifications. Van Nostrand Reinhold Co. N.Y. Text- vol: 970. Pl- Vol $212 p+847$.

Marchant, M. 1993. Foraminíferos de la Bahía Scholl, Región Magallánica, Chile, (Protozoa: Foraminifera). Gayana Zool. 57 (1): 61-75.

Murray, J.W. 1991. Ecology and Palaeoecology of Benthic Foraminifera.Longman, Harlow, $397 \mathrm{pp}$.

Murray, J. 2000. Ecology and applications Benthic Foraminifera. Cambridge Univ, Press 426 pp.
Violanti, D., B. Loi \& R. Melis 2000. Distribution of Recent Foraminifera from the Strait of Magellan. First quantitative data. Boll. Mus. Reg. Sci. Nat. Torino 17 (2): 511-539

Zapata, J. A. \& R. Alarcón 1988. Foraminíferos bentónicos del estrecho de Magallanes (52³3’S; 6954'W), Chile. Biota 4: 17-29. Osorno, Chile.

Zapata, J. \& H. Moyano 1997. Foraminíferos bentónicos recientes de Chile austral. Boletín Sociedad Biología 68: 27-37 Concepción, Chile.

Zapata, A. \& S. Varela 1975. Foraminíferos litorales recientes de bahía Maullín (413's; $\left.73^{\circ} 40^{\prime} \mathrm{W}\right)$ Chile. Rev. Ciencia y Naturaleza (Ecuador) 16 (1): 14-24. 
